\title{
Italian standardization of the Apples Cancellation Test
}

\author{
Mauro Mancuso - S. Rosadoni - D. Capitani - W. L. Bickerton - G. W. Humphreys - A. De Tanti • \\ M. Zampolini - G. Galardi - M. Caputo - S. De Pellegrin - A. Angelini - B. Bartalini - M. Bartolo • \\ M. C. Carboncini - P. Gemignani $\cdot$ S. Spaccavento $\cdot$ A. Cantagallo $\cdot$ P. Zoccolotti $\cdot$ G. Antonucci
}

Received: 17 November 2014/ Accepted: 19 January 2015

(C) Springer-Verlag Italia 2015

\begin{abstract}
Hemispatial neglect due to right parieto-temporo-frontal lesions has a negative impact on the success of rehabilitation, resulting in poor functional gain. Recent research has shown that different types of neglect can impact in a different way on rehabilitation outcomes. The availability of a sensitive test, useful for distinguishing egocentric and allocentric forms of neglect, may be clinically important as all current clinical instruments fail to distinguish between these forms of disturbance, yet they differentially predict outcome. The Apples Test is a new instrument useful to evaluate both egocentric and allocentric forms of neglect. In order to establish Italian norms for this diagnostic instrument the test was administered to a
\end{abstract}

Electronic supplementary material The online version of this article (doi:10.1007/s10072-015-2088-2) contains supplementary material, which is available to authorized users.

M. Mancuso $(\bowtie) \cdot$ S. Rosadoni · D. Capitani

Neurological Rehabilitation Unit, USL 9 Grosseto,

Grosseto, Italy

e-mail:m.mancuso62@gmail.com

W. L. Bickerton

School of Psychology, University of Birmingham,

Birmingham, UK

G. W. Humphreys

Department of Experimental Psychology, University of Oxford,

Oxford, UK

A. De Tanti

Centro Cardinal Ferrari, Fontanellato, Parma, Italy

M. Zampolini

Rehabilitation Department, USL2 Umbria, Umbria, Italy

G. Galardi

Rehabilitation Foundation Institute San Raffaele Giglio,

Cefalu, Palermo, Italy sample of 412 healthy people of both genders (201 M and $211 \mathrm{~F}$ ), aged from 20 to 80 years enrolled from 14 different rehabilitation centers in Italy. Based on the data, we established pathological performance cut-offs for the accuracy score (total omission errors), the asymmetry score for egocentric neglect (omission error difference), the asymmetry score for allocentric neglect (commission error difference) and execution time. The usefulness of the Apples Test for diagnostic purposes is illustrated by presenting three patients with different forms of neglect (egocentric, allocentric and mixed neglect).

Keywords Neglect - Egocentric neglect - Allocentric neglect · Assessment . Apple Test

M. Caputo

Neurological Rehabilitation Unit Auxilium Vitae,

Volterra, Pisa, Italy

S. De Pellegrin

Neuroscience Department, University of Padova, Padua, Italy

A. Angelini

IRCCS Foundation Don Carlo Gnocchi, Florence, Italy

B. Bartalini

Rehabilitation Department, USL12 Viareggio, Lucca, Italy

M. Bartolo

IRCCS Neurologic Institute Mediterraneo Neuromed, Pozzilli, Isernia, Italy

M. C. Carboncini

Neuro-rehabilitation Unit, Azienda Ospedaliera Universitaria

Pisana, Pisa, Italy 


\section{Introduction}

Understanding unilateral spatial neglect (USN) is crucial because of its functional implications [1] and impact on rehabilitation outcomes [2]. Indeed, its effects can persist long after the occurrence of stroke [3]. Reports of the rate of USN vary greatly in stroke research, ranging from about $15-80 \%$. These variations are presumably due to the different methods or tests used to carry out the assessments [4]. To limit the negative effects of neglect on patients' rehabilitation and functional recovery, a reliable assessment of neglect should be made early to begin appropriate treatment and monitor patients' recovery.

It has been argued that there are different types of neglect $[5,6]$. Here, we were particularly interested in the egocentric and allocentric forms of the disturbance, for which differential functional correlates have been found [7]. In particular, cases of double dissociation have been reported, with some patients neglecting objects only with respect to egocentric coordinates and other patients neglecting the left side of targets with respect to coordinates centered on the object but independent of the side of space $[8,9]$. However, it must be added that most patients show mixed forms of egocentric and allocentric neglect $[10,11]$. Thus, it is important to reliably assess individual performance. In this respect, it must be considered that standard tests of neglect in validated batteries [e.g., 12] do not allow distinguishing between these two forms of exploratory disturbance.

A useful instrument for this purpose is the Apples Test [13] which is specifically aimed at differentiating between allocentric (object-centered) and egocentric (stimuli-centered) forms of neglect as well as detecting general visual inattention. There is already some evidence of the clinical usefulness of this instrument, as it was validated with the Star Cancellation test [14] and recently standardized for the English population [15]. In view of the lack of clinically

P. Gemignani

Rehabilitative Foundation Don C. Gnocchi, Sarzana,

La Spezia, Italy

S. Spaccavento

Neurologic Unit, IRCCS Fondazione Maugeri, Cassano Murge, Bari, Italy

\section{A. Cantagallo \\ BrainCare, Padua, Italy}

P. Zoccolotti · G. Antonucci

Department of Psychology, Sapienza University of Rome, Rome, Italy

P. Zoccolotti · G. Antonucci

Neuropsychology Centre, IRCCS Santa Lucia Foundation,

Rome, Italy validated tests for distinguishing between egocentric and allocentric forms of neglect the availability of a sensitive test could prove clinically important.

In this study, we aimed to establish Italian norms for the clinical use of the Apples Test in this country. We also examined the correlations between gender, education, age and visuospatial attention tendencies. Three illustrative case studies are also provided; they demonstrate the different neglect performances identified by the Apples Test.

\section{Materials and methods}

\section{Sample}

Fourteen different rehabilitation centers in different parts of Italy participated in the study. A total of 412 healthy controls of both genders (201 M and $211 \mathrm{~F}$ ), aged between 20 and 80 years, participated in the study.

The following exclusion criteria were adopted:

- signs of previous (or ongoing at the time of the study) neurological and/or psychiatric conditions;

- left-handedness, as assessed by the Edinburgh Handedness Inventory [16];

- signs of cognitive impairment, as indicated by an MMSE score lower than 24/30 [17]: mean raw score was 29.02 (SD 1.30) and mean corrected score, 26.71 (SD 1.08);

- visual field defect revealed during a clinical examination.

The sample was stratified into three schooling levels (middle school, high school and college) and eight, tenyear age levels. The actual figures of the sample are presented in "Appendix".

The study was approved by the Ethical Committee of the coordination centre (Neurological Rehabilitation Unit, USL 9, Grosseto). All subjects signed a consent form before participating in the study.

Test

We used Bickerton et al. [13] original version of the Apples Test. This is a cancellation task in which outline drawings of 150 apples are shown pseudorandomly scattered over a sheet of A4 paper presented in a landscape orientation. All of the apples are presented in an upright position. One-third of the apples are full (targets) and twothirds are open on either the left or the right side (distractors). The test is presented in Fig. 1.

In order to balance the probability that omissions will show left versus right or upper versus lower space neglect, the page is divided into a grid with two rows and five columns to ensure an equal distribution of the apples across 




Fig. 1 The Apples Test

the page. The participants are unable to see the grid. Each cell of the grid contains 15 apples: three large ones (one with no opening, one with an opening on the left and one with an opening on the right) and 12 small apples (four without openings, four with openings on the left and four with openings on the right). The large apples are $50 \%$ bigger than the small apples. The midline of the page is positioned at the subjects' midline.

\section{Procedure}

Each participant was asked to cross out all the full apples and to ignore all the ones with holes. To ensure that the subjects understood the task instructions, a practice run-in task was given before the test was administered. This consisted of a mixture of targets and distractor items displayed only along the midline of the page (see Fig. 1).

A maximum of $5 \mathrm{~min}$ was allowed for completion of the test. The time taken to complete the task was recorded with a stopwatch.

\section{Scoring}

In accordance with the original version of the test, we considered the accuracy score as the total number of crossed-out targets (maximum 50). The asymmetry score for egocentric neglect is the difference between the number of targets selected on the right side in boxes 1-4 and the number of targets selected on the left side in boxes 7-10, excluding the upper and the lower box of the middle column (numbers 5 and 6). The maximum score is 20. Positive values indicate that more targets were selected on the right than on the left side (left neglect) and negative values indicate the opposite (right neglect).

The asymmetry score for allocentric neglect corresponds to the difference between the total number of distractor apples cancelled with a left opening and the number of distractors cancelled with a right opening (total left openings minus total right openings). Positive values indicate left neglect and negative values indicate right neglect.

Statistical analyses

Only complete data were analyzed using SPSS 18.1. Preliminary analyses indicated the absence of a significant effect of gender for all dependent variables (all ts $>1$, $p>0.4$ ); therefore, this variable was not analyzed further. Then, linear regression models were made to examine the relationship between the independent (age and schooling) and the dependent variables (omission errors, left-right error difference, commission errors and total time). As expected, there was a moderate negative correlation between age and years of schooling $(r=-0.16$; $p=0.001$ ), indicating a statistically redundant effect on the analyses. There was no significant correlation between age and schooling for any of the dependent variables. If the regression was not significant $(p>0.05)$ the cut-off values for the confidence interval to distinguish between a pathological and a normal performance were established (with a $95 \%$ confidence interval). If the regression was significant $(p<0.05)$, a conversion table was generated adjusting the expected values based on the influence of the independent variables.

Then, separately for each dependent measure on the Apples Test, we calculated the cut-off values of 
pathological performance as the value marking the $95 \%$ confidence limit.

\section{Results}

Accuracy score-total omission errors

Before proceeding with the statistical elaboration of data we checked for the possible presence of outliers in terms of total omission errors (defined as individual performances exceeding +3 with respect to a standardized mean). Based on this pre-analysis, data from eight subjects were excluded.

The linear regression model indicated the absence of any significant effect of the variables age and schooling on the omission errors $\left(F=1.67 ; \quad d f=2 \quad 391 ; r^{2}=0.008\right.$; $p=0.19)$. Therefore, the sample was pooled and we calculated the cut-off on all 404 subjects to differentiate normal from pathological performance. Mean total omission errors were 1.32 ( $\mathrm{SD}=1.68$; range from 0 to 7 ). Based on a $95 \%$ confidence limit $(1.32+1.96 \times 1.68=4.61)$ and calculating in excess, a cut-off of 5 was obtained. Therefore, a performance showing 6 or more (left + right) omission errors should be considered pathological.

Asymmetry score for egocentric neglect-omission error difference

Seven outliers were individuated in terms of the omission error difference (left minus right omission errors). The linear regression model indicated the absence of any significant effect of the variables age and years of schooling on the omission error difference $(F=0.186 ; d f=2392$; $\left.r^{2}=0.001 ; p=0.830\right)$. Therefore, we calculated the pathological performance cut-off for the total group of 405 subjects. The mean omission error difference was -0.03 $(\mathrm{SD}=0.905$; range from -3 to +3$)$. Based on a $95 \%$ confidence limit $(0.03+1.96 \times 0.905=1.74)$ and calculating in excess, a cut-off of 2 was obtained. Therefore, performances in which the difference between left and right total omissions is equal or above 3 should be considered pathological.

Asymmetry score for allocentric neglect-commission error difference

Commission errors occurred when subjects erroneously crossed out open apples. As in the previous cases, we looked for the presence of outliers (four in this case). The linear regression model indicated the absence of any significant effect of the variables age and schooling on the omission error difference score $\left(F=0.165 ; d f=2395 ; r^{2}=0.001\right.$; $p=0.848$ ). Therefore, we calculated the pathological performance cut-off on the total group of 408 subjects. The mean of the difference in the number of apples crossed out with a left opening minus the number of apples crossed out with a right opening was 0.021 ( $\mathrm{SD}=0.330$; range $0-3)$. Based on a $95 \%$ confidence limit $(0.021+1.96 \times 0.330$ $=0.668$ ) and calculating in excess, a cut-off of 1 was obtained. Therefore, performance in which the number of apples crossed out with a left opening minus the number of apples crossed out with a right opening was equal or above 2 should be considered as pathological. Positive numbers indicate right allocentric neglect and negative numbers indicate left allocentric neglect.

\section{Execution time}

We calculated the total time needed by the subjects to carry out the cancellation task. In this case, six subjects were considered outliers. Thus, the analyses were based on data from 406 subjects. The linear regression model indicated the presence of a significant effect of age and schooling variables on execution time $(F=16.69 ; d f=2392$; $\left.r^{2}=0.074 ; p=0.000\right)$. Based on this analysis, we obtained the following conversion formula:

Expected time $=84.76+0.58 \times$ age $+(-0.764) \times$ years of schooling,

which was used to develop a table with the expected execution times based on age and schooling (see Table 1). Then, we calculated the maximum time (beyond which performance can be considered as pathological) using the following formula:

Maximum time $=$ expected time $+1.96 \times \mathrm{SD}$ of residuals (36.42).

The maximum times as a function of age and schooling are also reported in Table 1.

\section{Comments}

Healthy subjects' performance showed a very high degree of accuracy although the stimuli are set out in relatively dense arrays. Indeed, as the subjects made very few omissions, 5 omissions (or more) can be considered a deviant explorative performance. The percentage of commission errors was even lower; thus, any value above 1 should be considered as pathological.

Overall, the availability of normative values for the Italian population make it possible to use the Apples Test not only as a research tool but also as a diagnostic instrument. To illustrate this, we briefly describe three patients who showed different performance profiles on the test, indicating different forms of neglect. 
Table 1 Expected and maximum time of execution in seconds

\begin{tabular}{|c|c|c|c|c|c|c|c|c|c|c|c|c|c|}
\hline \multirow[t]{2}{*}{ Education } & \multicolumn{13}{|l|}{ Age } \\
\hline & 20 & 25 & 30 & 35 & 40 & 45 & 50 & 55 & 60 & 65 & 70 & 75 & 80 \\
\hline \multicolumn{14}{|c|}{ Expected time } \\
\hline 5 & 92.4 & 95.3 & 98.2 & 101 & 104 & 107 & 110 & 113 & 115 & 118 & 121 & 124 & 127 \\
\hline 8 & 90.2 & 93 & 95.9 & 98.8 & 102 & 105 & 107 & 110 & 113 & 116 & 119 & 122 & 125 \\
\hline 13 & 86.3 & 89.2 & 92.1 & 95 & 97.9 & 101 & 104 & 106 & 109 & 112 & 115 & 118 & 121 \\
\hline 16 & 84 & 86.9 & 89.8 & 92.7 & 95.6 & 98.4 & 101 & 104 & 107 & 110 & 113 & 116 & 119 \\
\hline 18 & 82.5 & 85.4 & 88.3 & 91.2 & 94 & 96.9 & 99.8 & 103 & 106 & 108 & 111 & 114 & 117 \\
\hline 23 & 78.7 & 81.6 & 84.5 & 87.3 & 90.2 & 93.1 & 96 & 98.9 & 102 & 105 & 107 & 110 & 113 \\
\hline \multicolumn{14}{|c|}{ Maximum time } \\
\hline 5 & 164 & 167 & 170 & 172 & 175 & 178 & 181 & 184 & 187 & 190 & 193 & 196 & 198 \\
\hline 8 & 162 & 164 & 167 & 170 & 173 & 176 & 179 & 182 & 185 & 187 & 190 & 193 & 196 \\
\hline 13 & 158 & 161 & 163 & 166 & 169 & 172 & 175 & 178 & 181 & 184 & 187 & 189 & 192 \\
\hline 16 & 155 & 158 & 161 & 164 & 167 & 170 & 173 & 176 & 178 & 181 & 184 & 187 & 190 \\
\hline 18 & 154 & 157 & 160 & 163 & 165 & 168 & 171 & 174 & 177 & 180 & 183 & 186 & 188 \\
\hline 23 & 150 & 153 & 156 & 159 & 162 & 164 & 167 & 170 & 173 & 176 & 179 & 182 & 185 \\
\hline
\end{tabular}
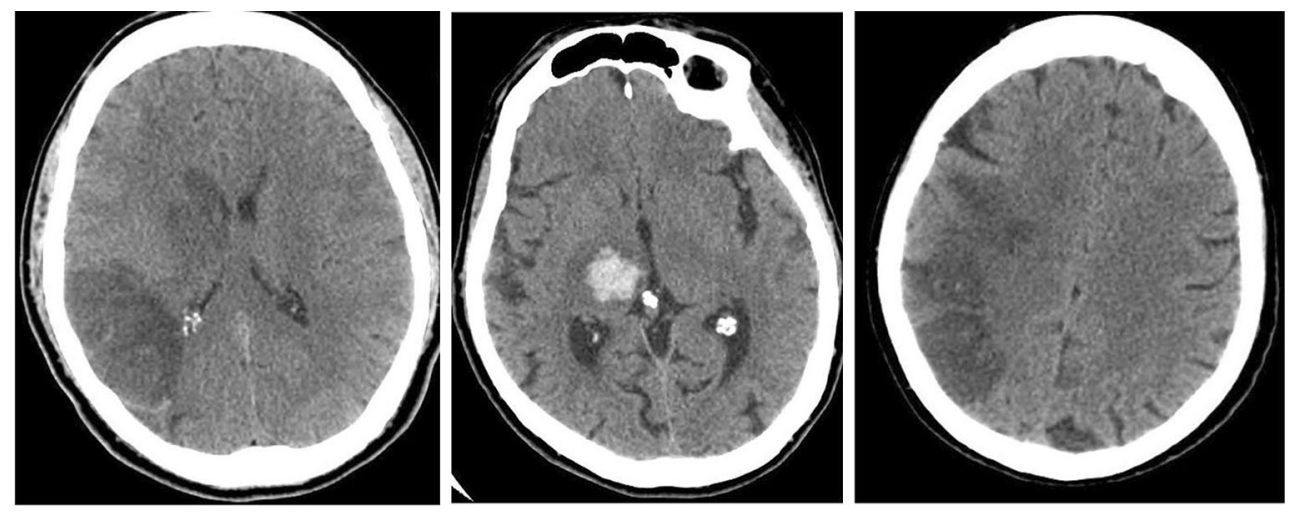

N.CA.

C.MC

Fig. 2 Illustrative CT scan images for V.ID., N.CA. and C.MC

\section{Illustrative cases}

Case V.ID.: egocentric neglect

V.ID., a 61-year-old retired married man with 13 years of schooling was referred to the Neurological Rehabilitation Unit in Grosseto with sequelae of a right cerebral ischemia. CT scan (see Fig. 2) indicated lacunar infarcts, LACI, according to Bamford et al's classification [18]. In his pre-morbid condition, the patient had full autonomy (pre-morbid Barthel index $=100 / 100$ ). After the event, he showed left hemiplegia with no trunk control (TCT $=61 / 100)$ [19]. Upright stance and ambulation were impossible (FIM = 64/126). He was dependent in daily life activities (ADL $=46 / 55)$.

On the Apples Test (see Fig. 3), his accuracy was very low (total omission score $=40$; cut-off $\geq 6$ ), but execution time was within normal limits $(157 \mathrm{~s}$; cut-off $=185 \mathrm{~s})$. V.ID. marked no stimuli on the left and his omission error difference (10) was well above the cut-off, i.e., $\geq 2$. V.ID. made no commission errors; therefore, both his total commission error score and his asymmetry commission error score were 0 (below the cut-offs of $\geq 2$ and $\geq 2$, respectively).

V.ID. appears to have severe egocentric neglect with an absence of allocentric neglect.

Case N.CA.: allocentric neglect

N.CA., a 73-year-old retired widow with 5 years of schooling was referred to the Neurological Rehabilitation Unit in Grosseto with sequelae of a right thalamic cerebral hemorrhagic lesion (see Fig. 2). Her pre-morbid condition 


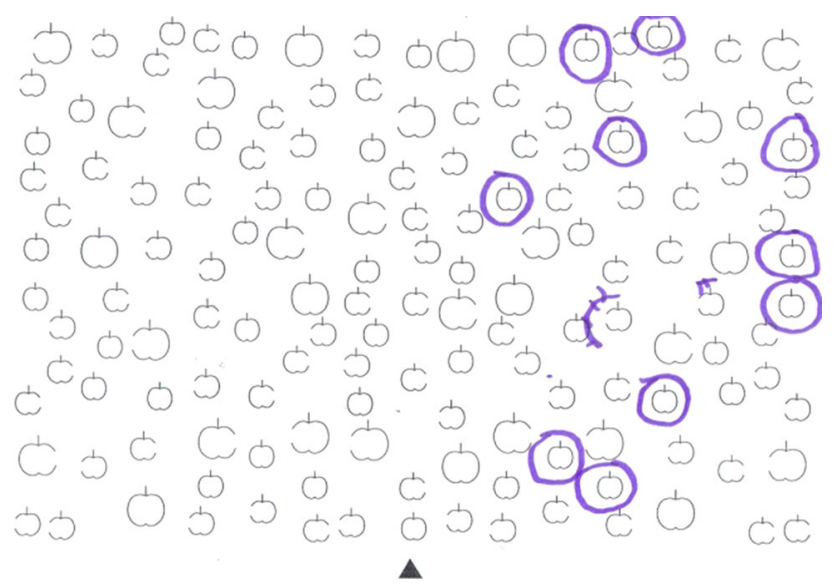

Fig. 3 Performance of V.ID. on the Apples Test. Note the presence of omissions and the absence of commission errors (egocentric neglect)

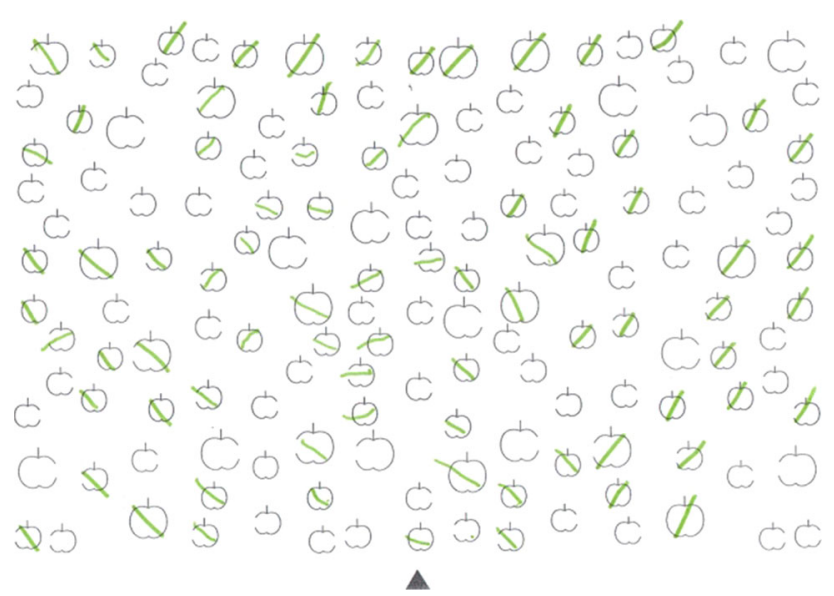

Fig. 4 Performance of N.CA. on the Apples Test. N.CA. made several commission errors on the left but made only one omission error (allocentric neglect)

was that of full autonomy (pre-morbid Barthel index $=100 / 100)$. At the evaluation she showed left hemiparesis with good trunk control $(\mathrm{TCT}=100)$; upright posture was possible but deambulation was compromised $(\mathrm{FIM}=56 / 126)$. She was dependent in daily life activities $(\mathrm{ADL}=44 / 55)$.

On the Apples Test (see Fig. 4) her accuracy score was within the limits (total omission score $=1$; cut-off $\geq 6$ ) but she was moderately slow (execution time $=213 \mathrm{~s}$; cutoff $=193$ ). Also, her score for egocentric neglect was within the normal limits (omission error difference $=1$; cut-off $\geq 2$ ). N.CA. made several commission errors $(N=30)$ that were well above the cut-off $(\geq 2)$. All commission errors were made on the left (asymmetry commission errors $=30$; cut-off $\geq 1$ ), indicating the presence of allocentric neglect but no sign of egocentric neglect.

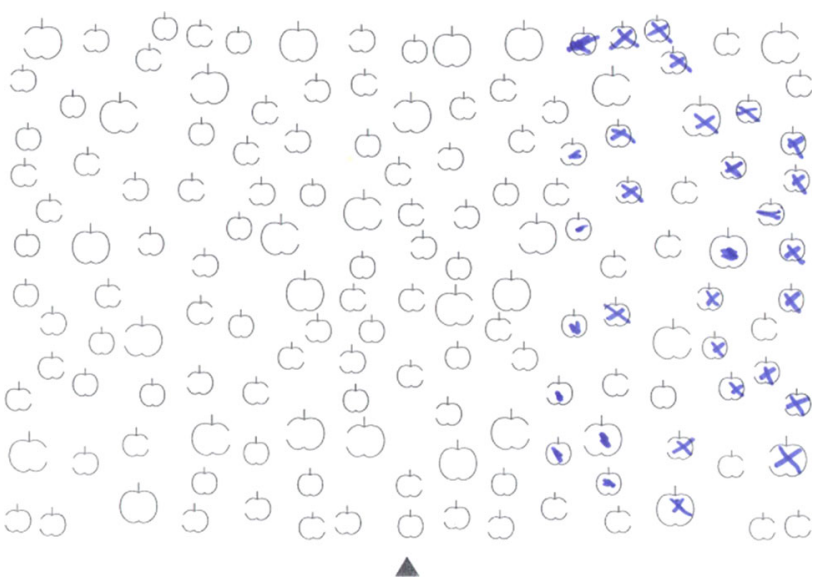

Fig. 5 Performance of C.MC. on the Apples Test. Note the presence of both omissions and commission errors indicative of a mixed disorder (allocentric and egocentric)

Case C.MC.: mixed neglect

C.MC., a 77-year-old retired widow with 8 years of schooling, was referred to the Neurological Rehabilitation Unit in Grosseto with sequelae of a right cerebral ischemia, LACI according to the Bamford et al. classification. Her pre-morbid condition was of full autonomy (pre-morbid Barthel Index $=100 / 100)$. After the event, she showed good trunk control (TCT $=61 / 100)$ but was unable to stand upright and walk (FIM $=61 / 126)$. She was dependent in daily life activities (ADL $=46 / 55$ ).

On the Apples Test (see Fig. 5) her performance was inaccurate (total omission score $=13$; cut-off $\geq 6$ ) but only moderately delayed (execution time $=197 \mathrm{~s}$; cutoff $=193$ ). She entirely neglected to mark stimuli on the left, thus obtaining a severely impaired omission error difference of 35 (cut-off $\geq 2$ ), indicating egocentric neglect. Also, her total number of commission errors (total commission errors $=16$; cut-off $\geq 2$ ) as well as her asymmetry commission errors $=16$; cut-off $\geq 1$ ) were pathological, indicating allocentric neglect.

As her performance was pathological in terms of both egocentric and allocentric neglect, C.MC. seems to have a mixed exploratory disorder.

\section{Discussion}

Neglect is a frequent symptom after unilateral brain damage and has many consequences on patients' daily activities and the effectiveness of motor rehabilitation [2, 20]. Much research has shown that neglect is segregated into different forms, such as neglect for personal versus extrapersonal space or neglect for near (within reaching) versus far (out of reaching) space. In the present study we focused 
on the contrast between extrapersonal allocentric and egocentric neglect, which are two forms of the disturbance and have distinct anatomical substrates [8, 21, 22]. Although egocentric neglect can be easily revealed by means of cancellation tests, no presently available clinical instruments are able to distinguish between the two forms of neglect. Thus, the Apples Test [13] is an important improvement over other currently available clinical instruments. The availability of normative values for the Italian population will allow accurately categorizing individual performance with respect to patients' age, gender and education.

The individual case studies presented here provide a cross section of possible performance profiles. V.ID. presented one form of egocentric neglect in the absence of any indication of allocentric neglect, whereas N.CA. showed the opposite pattern, i.e., pure allocentric neglect. Patients such as N.CA. are not in keeping with the idea that allocentric neglect necessarily co-exists with egocentric neglect, as proposed by some authors [10, 11]. However, it is probably true that mixed cases (such as C.MC.) are more frequent than pure egocentric or allocentric cases.

Overall, a sensitive diagnostic tool, such as the Apples Test, may be particularly useful for discriminating and describing different forms of exploratory disturbances in a clinical setting, thus allowing for better planning of the care management of patients.

Acknowledgments We would like to especially thank M. Pacini, B. Basagni, E. Todeschini, R. Moretti, S. Prestigiacomo, M.A. D'angelo, A. Lazzarini, C. Zucchella, M.E. Girò, F. Cellamare, E.Conti, M. Marchetti and G. Alla for their contribution in collecting the data.

Conflict of interest The authors declare that they have no conflicts of interest.

\section{Appendix}

See Table 2

Table 2 Number of healthy subjects who were part of the stratification sample as a function of age, gender and education

\begin{tabular}{|c|c|c|c|c|c|c|c|c|c|c|c|c|}
\hline \multirow[t]{3}{*}{ Education } & \multicolumn{12}{|c|}{ Age } \\
\hline & \multicolumn{2}{|c|}{$20-30$} & \multicolumn{2}{|c|}{$31-40$} & \multicolumn{2}{|c|}{$41-50$} & \multicolumn{2}{|c|}{$51-60$} & \multicolumn{2}{|c|}{$61-70$} & \multicolumn{2}{|c|}{$71-80$} \\
\hline & $\mathrm{M}$ & $\mathrm{F}$ & $\mathrm{M}$ & $\mathrm{F}$ & $\mathrm{M}$ & $\mathrm{F}$ & M & $\mathrm{F}$ & M & $\mathrm{F}$ & M & $\mathrm{F}$ \\
\hline$\leq 8$ & 5 & 7 & 10 & 8 & 12 & 13 & 11 & 14 & 11 & 13 & 11 & 12 \\
\hline $9-13$ & 13 & 12 & 14 & 12 & 13 & 16 & 14 & 13 & 12 & 14 & 8 & 6 \\
\hline$\geq 14$ & 12 & 15 & 13 & 15 & 12 & 13 & 14 & 13 & 9 & 9 & 7 & 6 \\
\hline
\end{tabular}

\section{References}

1. Jehkonen M, Ahonen JP, Dastidar P, Koivisto AM, Laippala P, Vilkki J, Molnar G (2000) Visual neglect as a predictor of functional outcome one year after stroke. Acta Neurol Scand 101(3):195-201

2. Paolucci S, Antonucci G, Grasso MG, Pizzamiglio L (2001) The role of unilateral spatial neglect in rehabilitation of right braindamaged ischaemic strple patients: a matched comparison. Arch Phys Med Rehab 82:743-749

3. Nijober TCW, Kollen BJ, Kwakkel G (2013) Time course of visuospatial neglect early after stroke: a longitudinal cohort study. Cortex 49:2021-2027

4. Bowen A, McKenna K, Tallis RC (1999) Reasons for variability in the reported rate of occurrence of unilateral spatial neglect after stroke. Stroke 30:1196-1202

5. Milner DA, McIntosh RD (2005) The neurological basis of visual neglect. Curr Opin Neurol 18:748-753

6. Bartolomeo P (2007) Visual neglect. Curr Opin Neurol 20:381-386

7. Chechlacz M, Rotshtein P, Roberts KL, Bickerton W-L, Lau JKL, Humphreys GW (2012) The prognosis of allocentric and egocentric neglect: evidence from clinical scans. PLoS One 7(11):e47821. doi:10.1371/journal.pone.0047821

8. Grimsen C, Hildebrandt H, Fahle M (2008) Dissociation of egocentric and allocentric coding of space in visual search after right middle cerebral artery stroke. Neuropsychologia 46:902-914

9. Ota H, Fujii T, Suzuki K, Fukatsu R, Yamadori A (2001) Dissociation of body centered and stimulus centered representation in unilateral neglect. Neurology 57:2064-2069

10. Rorden C, Hjaltason H, Fillmore P, Fridriksson J, Kjartansson O, Magnusdottir S, Karnath HO (2012) Allocentric neglect strongly associated with egocentric neglect. Neuropsychologia 50:1151-1157

11. Yue Y, Song W, Huo S, Wang M (2012) Study on the occurrence and neural bases of hemispatial neglect with different reference frames. Arch Phys Med Rehabil 93:156-162

12. Stone SP, Wilson B, Wroot A, Halligan PW, Lange LS, Marshall JC, Greenwood RJ (1991) The assessment of visuo-spatial neglect after acute stroke. J Neurol Neurosurg Psychiat 54:345-350

13. Bickerton WL, Samson D, Williamson J, Humphreys GW (2011) Separating forms of neglect using the Apple Test: validation and functional predicting in chronic and acute stroke. Neuropsychology 25:567-580

14. Wilson B, Cockburn J, Halligan P (1987) Development of a behavioural test of visuospatial neglect. Arch Phys Med Rehabil 68:98-102

15. Humphreys G, Bickerton WL, Samson D, Riddoch M (2012) BCoS cognition screen. Psychology Press, Hove

16. Oldfield RC (1971) The assessment and analysis of handedness: the Edinburgh inventory. Neuropsychologia 9:97-113

17. Magni E, Binetti G, Bianchetti R, Rozzini R, Trabucchi M (1996) Mini-Mental State Examination: a normative study in Italian elderly population. Eur J Neurol 3:198-202

18. Bamford J, Sandercock P, Dennis M, Warlow C, Burn J (1991) Classification and natural history of clinically identifiable subtypes of cerebral infarction. The Lancet 337:1521-1526

19. Collin C, Wade DT (1990) Assessing motor impairment after stroke: a pilot reliability study. J Neurol Neurosurg Psychiat 53:576-579

20. Denes G, Semenza C, Stoppa E, Lis A (1982) Unilateral spatial neglect and recovery from hemiplegia a follow-up study. Brain 105(3):543-552 
21. Chechlacz M, Rotshtein P, Bickerton WL, Hansen PC, Deb S, Humphreys GW (2010) Separating neural correlates of allocentric and egocentric neglect: distinct cortical sites and common white matter disconnections. Cogn Neuropsychol 27:277-303
22. Medina J, Kannan V, Pawlak MA, Kleinman JT, Newhart M, Davis C, Heidler-Gary JE, Herskovits EH, Hillis AE (2009) Neural substrates of visuospatial processing in distinct reference frames: evidence from unilateral spatial neglect. J Cogn Neurosci 21:2073-2084 